\title{
Assessing Anticoagulation Management and Shared Decision-Making Documentation from Providers Participating in the SUPPORT-AF Study
}

\section{Laboni Hoque}

University of Massachusetts Medical School

\section{Azraa Amroze}

University of Massachusetts Medical School

\section{Vinit Gilvaz}

St. Vincent Hospital

\section{Sonu Abraham}

St. Vincent Hospital

\section{Amos Lal}

Mayo Clinic Minnesota

\section{Ajay Mishra}

St. Vincent Hospital

\section{Sybil Crawford}

University of Massachusetts Medical School

\section{Kathleen Mazor}

University of Massachusetts Medical School

\section{David McManus}

University of Massachusetts Medical School

Alok Kapoor ( $\sim$ alok.kapoor@umassmemorial.org )

University of Massachusetts Medical School https://orcid.org/0000-0003-1300-7124

\section{Research article}

Keywords: atrial fibrillation, anticoagulation, shared decision-making, academic detailing

Posted Date: August 19th, 2019

DOI: https://doi.org/10.21203/rs.2.13133/v1

License: (1) (1) This work is licensed under a Creative Commons Attribution 4.0 International License.

Read Full License 
Version of Record: A version of this preprint was published at Journal of Continuing Education in the Health Professions on January 1st, 2020. See the published version at https://doi.org/10.1097/CEH.0000000000000293. 


\section{Abstract}

\section{Background}

We previously tested an intervention featuring educational outreach with modified academic detailing $(A D)$ to increase anticoagulation $(A C)$ use in patients with atrial fibrillation (AF). Currently, we compare providers receiving and not receiving $A D$ in terms of inclusion of our $A D$ educational topics and shared decision-making elements in documentation.

\section{Methods}

Physicians reviewed themes discussed with providers during AD and evaluated charts for evidence of shared decision-making. We then compared frequency of documentation of individual items for providers receiving $A D$ compared with non-AD providers. To understand baseline documentation practices of $A D$ providers, we also randomly selected encounters of $A D$ providers before their $A D$ participation.

\section{Results}

There were 113 eligible encounters in the four months after AD - 36 from AD providers and 77 from non$A D$ providers. We also identified 35 encounters from $A D$ providers before participating in the intervention. Providers infrequently documented many reviewed items (\% documenting): AC mentioned (44\%), multiple options for AC (5\%), CHA2DS2-VASc score (11\%), bleeding risk factors (2\%). Compared with non-AD providers, $A D$ providers had statistically significant higher percentages for the following items: mention of AC (64\% versus $35 \%)$, stroke risk ( $11 \%$ versus $0 \%)$, AC benefits ( $8 \%$ versus $0 \%)$, patient involvement (70\% versus $0 \%$ ). There was no improvement, however, for $A D$ providers compared with baseline documentation percentages.

\section{Conclusions}

Providers infrequently documented important items in AC management and shared decision-making. AD participation did not improve documentation. Improving adoption of AD educational items may require more prolonged interaction with providers. Improving shared decision-making may require an intervention more focused on it and its documentation.

\section{Trial Registration}

The trial was registered as NCT03583008 on Clinicaltrials.gov on July 11, 2018.

\section{Background}

Anticoagulation $(\mathrm{AC})$ therapy is the cornerstone for stroke prevention in patients with atrial fibrillation (AF). However, only about half of eligible patients receive AC in US-based, ambulatory registries. ${ }^{1}$ There are multiple factors that likely contribute to the gap in $\mathrm{AC}$ use. Healthcare providers often over-estimate 
risks and seriousness of bleeding from $A C$ and under-estimate risks of stroke from $A C$ undertreatment in their AF patients. ${ }^{1}$ At the same time, patients may be reluctant to initiate or continue AC due to real or perceived risks of side effects, burdens from monitoring, and financial costs. ${ }^{2}$

Giving healthcare providers up-to-date evidence about use of AC may help to reduce undertreatment. In addition, giving providers information about direct oral anticoagulants (DOACs) may enable them to overcome patient hesitation to use AC based on prior poor experience/dissatisfaction with use of warfarin. In light of the complexity of the decision and the strong influence of patient preferences and knowledge of successful AC treatment, the American Heart Association/American College of Cardiology Atrial Fibrillation treatment guidelines recommend that healthcare providers use shared decision-making when advising AF patients about the risks and benefits of oral anticoagulation. ${ }^{3}$ Without full patient understanding of the risks and benefits, patients may fail to realize the value of the therapies providers suggest.

Traditional educational methods, such as continuing medical education (CME) and/or dissemination of treatment guidelines, have not proven effective in reducing the AC treatment gap. ${ }^{4}$ We recently completed a cluster randomized controlled trial comparing increase in $\mathrm{AC}$ use for providers receiving electronic messaging and educational outreach in the form of academic detailing (AD) with usual care. Approximately one third of providers participated in both parts of the intervention whereas another third received only electronic messaging. We did not see a statistically significant effect on AC use for the combined messaging $+A D$ intervention. Comparing documentation of encounters conducted by $A D$ providers to those conducted by non-AD providers may shed light into the $A C$ management and use of elements of shared decision-making.

We therefore conducted a comprehensive review of the documentation of patient encounters occurring up to four months after completion of AD. Our intent was to understand how often providers addressed AC for $A F$ in their documentation and how often their documentation reflected the various topics covered during our $A D$ sessions. We also reviewed their documentation for evidence of shared decision-making elements, including mention of options for treatment, mention of the risk of stroke, risk of bleeding, and evidence of involvement of the patient in discussion about AC.

\section{Methods}

This study was approved by the University of Massachusetts IRB.

Population and setting. We have previously described our population and setting. ${ }^{5}$ Briefly, we identified cardiology and primary care providers within the large health care system affiliated with our medical school in central Massachusetts. To be eligible, providers had to be taking care of at least 5 patients with $\mathrm{AF}$ who had an elevated stroke risk (i.e. $\mathrm{CHA}_{2} \mathrm{DS}_{2}$-VASc score $\geq 2$ ) and were not currently on $\mathrm{AC}$ using the repository of patients available from our electronic medical record (EMR). 


\section{Procedure:}

$A D$ intervention. Our $A D$ approach included two live sessions spaced 1-2 months apart beginning July 2018 and finishing November 2018. For each session, conducted either in person or remotely by web conferencing software, we reviewed those topics chosen by the provider from a list of 10 general topics. Themes included the role of aspirin in preventing strokes, AC use in the elderly, use of stroke and bleeding risk calculators, resuming $\mathrm{AC}$ after bleeding or fall, dual and triple antithrombotic therapy, treating a patient with depression and/or cognitive impairment, DOAC information, common scenarios where AC decision-making is complex, resources available to providers at our institution, and general resources. (Appendix A for presentation material) We followed up our first meeting by supplying providers with any evidence or articles that were discussed during the $A D$ session. We also sent one email reminding the provider about what we discussed and to prompt them to try to implement any practice changes that they had agreed to try after the first session. The second session was shorter and included a recap of the first session and dialogue about the provider's experience with AC management for patients seen in the interim between first and second sessions.

Chart review. We identified outpatient encounters of patients being seen by $A D$ and non-AD providers in the four months following AD (December 2018 until March 2019. Four physician reviewers were trained to conduct chart reviews. Each physician reviewer had completed at least one year of internal medicine residency. None of the physicians were aware of the treatment assignment of providers (i.e. they were blinded). During the training process, the 4 resident physicians reviewed charts until their responses were consistent with a faculty author (AK). Reviewers then each reviewed between 25 and 35 charts of the final analysis set. Reviewers responded to several items included in a chart review form developed for our study. In the form, we asked physicians to confirm the patient was not on AC at the time of the encounter. We also asked reviewers to determine if the visit was a regular, comprehensive visit versus a visit scheduled because of acute illness or other narrow purpose. Reviewers then evaluated if the patient started AC within three months of the visit. Next, reviewers assessed whether providers discussed any of the themes that we mentioned during our AD sessions. Finally, our reviewers evaluated charts for evidence of shared decision-making, including mention of options for treatment, mention of the risk of stroke such as documentation of the $\mathrm{CHA}_{2} \mathrm{DS}_{2}$-VASc score, risk of bleeding, and evidence of involvement of the patient in discussion about AC.

In addition to examining charts of encounters occurring after providers received $A D$, we also reviewed charts for this group of providers prior to beginning the intervention to gain additional insight into the impact of our intervention given that the providers who agreed to meet with us for AD were potentially different in terms of diligence with documentation compared with other providers.

Analysis. We compared the frequency of documentation of individual items in providers receiving AD compared with remaining providers. We also compared the frequency of each of the items in visits conducted by AD providers before versus after intervention. For all our comparisons, we used Fisher's exact test due to relatively small numbers we were analyzing for the comparisons. To consider a 
comparison statistically significant, we used a p-value $<0.05$. We conducted all analyses in Microsoft Excel 2016 (Microsoft, Inc. Redmond, WA).

\section{Results}

We identified 113 patients having encounters with 62 providers. Of these 113 encounters, we found that $22 \%$ of the time, patients started AC within three months of the index visit. Although more than $90 \%$ of the encounters were scheduled/comprehensive visits, AF was mentioned only $57 \%$ of the time. Linked to this finding is that providers discussed AC in only $44 \%$ of visits. Moreover, providers did not document having discussed multiple options with patients (only $5 \%$ of the time). They also infrequently documented the $\mathrm{CHA}_{2} \mathrm{DS}_{2}$-VASc score (11\% of the time). Similarly, providers infrequently documented discussion of the resumption of $A C$ after bleeding (although we did not establish how often a patient had a bleeding history for which the resumption of $A C$ was relevant). They also infrequently documented special topics ( $5 \%$ of the time) that were part of our $A D$ intervention.

Of the 113 encounters, 36 of these were with patients seeing one of 18 providers who received AD; 77 encounters included patients seeing one of 44 non-AD providers. We also identified 35 visits for patients seeing one of $17 A D$ providers prior to the provider receiving the intervention; 12 of these providers were also among the set of $18 \mathrm{AD}$ providers contributing encounters in the post-intervention phase.

There were several differences in documentation between encounters of $A D$ and non-AD providers. Most notably, in $64 \%$ of encounters with AD providers, there was mention of $A C$ vs. only $35 \%$ of encounters with non-AD providers ( $p$-value 0.005 ). In addition, $A D$ providers more frequently documented the risk of stroke ( $11 \%$ vs. $0 \%$ p-value 0.009 ). They also more frequently discussed the benefits of $A C$ and more frequently made reference to patient involvement in the discussions although in general both groups infrequently documented these items. (Table 1 for further details)

When we compared the frequency of documentation of most items in AD providers pre- vs. postintervention, we found multiple differences. Prior to intervention, $A D$ providers more frequently documented multiple AC options ( $29 \%$ vs. $6 \%$ p-value 0.012 ). Similarly, prior to intervention, AD providers more frequently documented discussing AC with their patients ( $40 \%$ vs. $17 \%$ p-value 0.037 ). Before intervention, they documented a trend towards (as opposed to a statistically significant difference) more patient involvement in the discussion ( $37 \%$ vs $17 \%$ p-value 0.064$)$. In contrast, post-intervention $A D$ providers more frequently documented risk of stroke ( $11 \% \mathrm{vs} 0 \% \mathrm{p}$-value 0.115$)$, more frequently addressed bleeding risk factors ( $6 \%$ vs $0 \% \mathrm{p}$-value 0.493 ), and more frequently documented one of the special topics from AD (14\% vs $3 \%$ p-value 0.199$)$.

\section{Discussion}

We found low rates of documentation of several elements of AC management and shared decisionmaking. This included low rates of documentation of stroke risk $\left(\mathrm{CHA}_{2} \mathrm{DS}_{2}-\mathrm{VASc}\right)$, bleeding risk, mention 
of multiple options for treatment, mention of timelines for resuming AC after bleeding, and special topics we discussed in our $A D$ sessions. Although $A D$ providers more frequently documented these elements compared with non-AD providers, this did not appear to reflect an improvement compared to review of documentation written by $A D$ providers prior to intervention.

$A D$ has been shown to successfully improve provider adherence to guidelines in a variety of circumstances including for blood pressure management of patients at high risk for cardiovascular disease, ${ }^{6}$ appropriate use of antibiotics among hospitalists, ${ }^{7}$ and AC prescription for uptake of DVT prophylaxis. ${ }^{8}$ In the context of stroke prevention for $A F$, previous investigators assessed written feedback and $A D$ sessions delivered among 66 providers over a 12-month period via telephone. They found no significant difference in $\mathrm{AC}$ prescription rates among 1,116 AF patients treated by providers receiving the intervention. ${ }^{9}$ We observed similar results in our SUPPORT-AF II study which we described above. To our knowledge no one has previously assessed the impact of $A D$ on quality of documentation of $A C$ management. Provider documentation often correlates with quality of patient care, particularly regarding continuity of care between providers. ${ }^{10}$ In the 2011 AMIA policy meeting, participants discussed ways to foster improved documentation practices with goal of reducing healthcare costs and improving quality. ${ }^{11}$ Therefore, assessing documentation of $\mathrm{AC}$ management is an important step in evaluating and potentially improving AC management.

The main implication of our findings is that $A D$ delivered using our approach of two meetings plus interim correspondence and provider support did not predict an increase in the frequency of documentation of elements of AC management, including shared decision-making. Improving the documentation of these items may require more prolonged interaction with providers. In addition, documentation of shared decision-making items may require an intervention more focused on documentation. Although professional societies have made recommendations to encourage shared decision-making with regard to $\mathrm{AC}$ for $\mathrm{AF}^{3,12}$ to our knowledge, they have not disseminated document templates/checklists for shared decision-making content or the organization of the content.

There are limitations to the findings we report. There is an unclear correlation between documentation of discussion with patients compared with what was actually discussed. Audio recordings would provide complete information but were not part of the original scope of our study. We welcome research looking at the conversations occurring between patients and providers and examining the correlation with provider documentation. In addition, there were some providers who participated in more than one of the encounters that we reviewed. Therefore, the effective sample size of our study was relatively modest.

Nevertheless, given the overall low rates of documentation of the items of interest, we do not believe the intervention as deployed would be successful in improving documentation in a larger sample.

\section{Conclusions}

Both $A D$ and non-AD providers infrequently documented several important factors in $A C$ management and shared decision-making. Participation in our AD intervention did not significantly improve the 
documentation of these elements. Based on these findings, we suspect that this method of AD alone is not sufficient to increase the frequency of documentation of these elements. For improving documentation of shared decision-making, we encourage research using a more focused intervention and the development of templates to facilitate shared decision-making documentation.

\section{Declarations}

\section{List of Abbreviations}

$A D=$ academic detailing; $A C=$ anticoagulation; $A F=$ atrial fibrillation

\section{Ethics Approval and Consent to Participate}

This study was approved by the University of Massachusetts IRB.

\section{Consent for Publication}

Not applicable

\section{Availability of Data and Materials}

The datasets used and/or analysed during the current study are available from the corresponding author on reasonable request.

\section{Competing Interests}

Dr. Kapoor has received research grant support from Pfizer through its Independent grants for learning and change the funding mechanism and from Bristol-Myers Squibb for Independent Medical Education Grants. More recently, he has received research grant support through a competitive process adjudicated and funded by the alliance, which is formed by both Pfizer and Bristol-Myers Squibb. He has also been awarded a grant by Pfizer to examine conversations between patients and providers.

Dr. McManus receives sponsored research support from Bristol Myers Squibb, Boeringher Inghelheim, Pfizer, Biotronik, Philips Healthcare, and has consulted for Bristol Myers Squibb, FlexCon, Samsung, Philips, and Pfizer. Dr. McManus has equity in Mobile Sense Technologies, LLC. Dr. McManus also reports funding from National Institutes of Health (R01HL137794-01A1 and R01HL126911-03).

All other investigators have declared that they do not have any conflicts of interest.

\section{Funding}


This study was funded by a grant through an Independent Medical Education funded by Bristol-Myers Squibb under the request ID 28358345.

\section{Authors' Contributions}

LH conducted chart reviews, analyzed data, and contributed to writing the manuscript. AA collected and analyzed data and made critical revisions to the manuscript. VG, SA, AL, and AM conducted chart reviews and made critical revisions to the manuscript. SC, KM, and DM made critical revisions to the manuscript. AK conducted chart reviews, analyzed data, and contributed to writing the manuscript. All authors read and approved the final manuscript.

\section{Acknowledgements}

Not applicable

\section{Table}

Due to technical limitations, Table 1 is only available for download from the Supplementary Files section.

\section{Supplementary Files}

This is a list of supplementary files associated with this preprint. Click to download.

- Table1.pdf

- CONSORT2010Checklistak.pdf

- APPENDIXA.pdf 